\title{
Stability and convergence analysis of multi-agent consensus with information reuse
}

\author{
Ziyang Meng ${ }^{\mathrm{ab}}$, Yongcan $\mathrm{Cao}^{\mathrm{b}}$ and Wei Ren ${ }^{\mathrm{b} *}$ \\ ${ }^{a}$ Department of Precision Instruments and Mechanology, Tsinghua University, Beijing, 100084, PR China; \\ ${ }^{b}$ Department of Electrical and Computer Engineering, Utah State University, Logan, UT 84341, USA
}

(Received 26 March 2009; final version received 24 December 2009)

\begin{abstract}
In this article, we study multi-agent consensus algorithms with information reuse by intentionally introducing the outdated state information into the traditional consensus algorithms. In the continuous-time case, we first show that the outdated state information combined with the current state information does not necessarily jeopardise the stability of a single system, but may improve the convergence speed without increasing the maximal control effort. Then this idea is extended from the single-agent case to the multi-agent case. When the directed communication graph is fixed, the corresponding Laplacian matrix and the outdated state information satisfy certain conditions, we show that the consensus algorithm with both the current and outdated states can achieve a faster convergence speed than the standard one. We also consider the case of a switching directed communication graph and derive corresponding conditions. In the discrete-time case, we propose a discrete-time consensus algorithm with both the current and outdated states under an undirected fixed communication graph. We then derive conditions on the communication graph, the sampling period and the outdated state information such that the proposed algorithm can achieve a faster convergence speed than that using the standard one. In both the continuous-time and discrete-time settings, we show that the maximum control efforts for the proposed consensus algorithms are identical to those for the standard ones. Several simulation examples are presented as a proof of concept.
\end{abstract}

Keywords: consensus; cooperative control; multi-agent systems; outdated state

\section{Introduction}

Multi-agent systems have received significant attention in recent years because various benefits, such as low complexity, high robustness and great efficiency can be achieved by having a group of agents work cooperatively. A fundamental approach used in multi-agent systems is consensus. Consensus means that agents in a communication network achieve an agreement on their common state by exchanging information with their neighbours, which is of significance in many applications, such as formation control (Fax and Murray 2004), rendezvous (Lin, Morse, and Anderson 2003), and attitude alignment (Ren 2007). Numerous results on stability and convergence of multi-agent consensus were obtained by using matrix analysis and graph theory.

One of the most important problems in consensus is that how the communication graph influences the stability of multi-agent systems. Motivated by Vicsek, Czirok, Jacob, Cohen, and Schochet (1995), the authors in Jadbabaie, Lin, and Morse (2003) showed that consensus can be achieved if the undirected communication graph is jointly connected.
The authors in Moreau (2005), Ren and Beard (2005) and Olfati-Saber and Murray (2004) extended the results in Jadbabaie et al. (2003) to the case of a directed communication graph. It was shown that consensus can be achieved if the directed switching communication graphs have a directed spanning tree jointly.

In addition to the stability analysis, another important feature of consensus algorithms is the convergence speed. The authors in Olfati-Saber and Murray (2004) studied the convergence speed of a consensus algorithm and showed that the convergence speed is determined by the second smallest eigenvalue of the Laplacian matrix. Moreover, a semi-definite programming-based approach was provided in Kim and Mesbahi (2006) to maximise the second smallest eigenvalue of a state-dependent Laplacian matrix such that the fastest convergence speed is achieved. The authors in Xiao and Boyd (2003) proposed an accurate definition of the convergence speed for consensus, and derived a linear iteration algorithm to find the optimal weight matrix for the communication graph in order to obtain the fastest convergence speed.

*Corresponding author. Email: wren@engineering.usu.edu 
Considering the fact that time delays are inevitable in real systems, consensus algorithms with time delay have been studied in different settings. The authors in Olfati-Saber and Murray (2004) studied a uniform input time delay under an undirected fixed communication graph while the authors in Lee and Spong (2006) discussed a nonuniform communication time delay under a general dynamic model. It was shown in Moreau (2004b) that the uniform communication time delay will not affect the stability of a consensus algorithm under directed fixed or switching communication graphs. The authors in Xiao and Wang (2008a, b) used nonnegative matrix theory to study, respectively, the continuous-time and discrete-time consensus algorithms with time-varying delays under a switching network topology. The authors in Tian and Liu (2008) provided a unified model considering both the communication and input delays and presented conservative conditions to guarantee the stability. In Peng and Yang (2009), a Lyapunov energy method was introduced to discuss the stability of the leader-follower consensus problem under time-varying delays. In these references, time delays were considered a disadvantage, which will affect the stability of the system. In contrast, the authors in Ghosh, Muthukrishnan, and Schultz (1998) modelled a diffusive schedule in a discrete-time setting, where outdated time-delayed state information was introduced intentionally. It was shown that the outdated state information combined with the current state information can be used to improve the convergence speed of the distributed load balancing algorithm. This approach was also adopted in Cao, Spielman, and Yeh (2006), where gossip algorithms were considered for a distributed averaging problem in a network, and in Cao and Ren (2009), where a consensus algorithm was discussed in a continuous-time setting under fixed and undirected interaction.

In this article, we first extend the existing results in Cao and Ren (2009) to the directed fixed and switching communication graphs in a continuous-time setting and derive conditions on the communication graphs and the outdated state information such that the consensus algorithm with both the current and outdated state information achieves a faster convergence speed than that using the standard one without increasing the maximum control effort. We show the significant difference between the cases of an undirected communication graph and that of a directed communication graph and obtain some interesting conclusions. We then give the corresponding stability and convergence analysis on the discrete-time case under an undirected fixed graph. The conditions on the communication graph, the sampling period and the outdated state information are given such that the proposed discrete-time consensus algorithm with both the current and outdated state information achieves a faster convergence speed than that using the standard one without increasing the maximum control effort.

This article is organised as follows. In Section 2, we introduce the graph theory notions used in this article. In Section 3, we analyse the continuous-time consensus algorithm with both the current and outdated states under the directed fixed and switching communication graphs. In Section 4, the stability and the convergence speed of the proposed discrete-time algorithm with both the current and outdated states are discussed under an undirected fixed communication graph. In Section 5, simulations are given to validate the theoretical results. The conclusion is given in Section 6.

\section{Graph theory notions}

Using graph theory, we can model the communication among agents in a multi-agent system. A directed graph $\mathcal{G}$ consists of a pair $(V, \mathcal{E})$, where $V$ is a finite nonempty set of nodes and $\mathcal{E} \in V \times V$ is a set of ordered pairs of nodes. An edge $\left(v_{i}, v_{j}\right)$ denotes that node $v_{j}$ can obtain information from $v_{i}$, but not necessarily vice versa. All neighbours of node $v_{i}$ are denoted as $N_{i}:=\left\{v_{j} \mid\left(v_{j}, v_{i}\right) \in \mathcal{E}\right\}$. An undirected graph $\mathcal{G}$ is defined such that $\left(v_{j}, v_{i}\right) \in \mathcal{E}$ implies $\left(v_{i}, v_{j}\right) \in \mathcal{E}$.

A directed path is a sequence of edges in a directed graph of the form $\left(v_{i_{1}}, v_{i_{2}}\right),\left(v_{i_{2}}, v_{i_{3}}\right), \ldots$ An undirected path in an undirected graph is defined analogously. A directed graph has a directed spanning tree if there exists at least one node having a directed path to all other nodes. An undirected graph is connected if there is an undirected path between every pair of distinct nodes.

The adjacency matrix $\mathcal{A}=\left[a_{i j}\right] \in \mathbb{R}^{n \times n}$ associated with $\mathcal{G}$ is defined such that $a_{i j}$ is a positive value if $\left(v_{j}, v_{i}\right) \in \mathcal{E}$ while $a_{i j}=0$ otherwise. The (nonsymmetric) Laplacian matrix $\mathcal{L}=\left[l_{i j}\right] \in \mathbb{R}^{n \times n}$ associated with $\mathcal{A}$ is defined as $l_{i i}=\sum_{j \neq i} a_{i j}$ and $l_{i j}=-a_{i j}$, where $i \neq j$. In addition, $\mathcal{L}$ has a simple zero eigenvalue with an eigenvector of $\mathbf{1}_{n}$, where $\mathbf{1}_{n}$ is the $n \times 1$ all-one vector, and all other eigenvalues have positive real parts, if and only if $\mathcal{G}$ has a directed spanning tree (Agaev and Chebotarev 2000).

\section{Continuous-time case}

In this section, we first study the single-agent case with a complex control gain because the eigenvalues of the Laplacian matrix $\mathcal{L}$ associated with a directed graph may be complex. Then the single-agent case is extended to the multi-agent case under the directed fixed and 
dynamic communication graphs. The conditions on the communication graph and the outdated state information are given such that the consensus algorithm with both the current and outdated states can achieve consensus with a faster convergence speed than that using the standard one without increasing the maximum control effort.

\subsection{Single-agent case}

For a single-agent system with single-integrator kinematics given by

$$
\dot{\varphi}(t)=u(t),
$$

where $\varphi \in \mathbb{C}$ is the state and $u \in \mathbb{C}$ is the control input, we study two different control algorithms as follows:

$$
\begin{gathered}
u(t)=2(a+\mathbf{j} b) \varphi(t), \\
u(t)=(a+\mathbf{j} b)[\varphi(t)+\varphi(t-\tau)],
\end{gathered}
$$

where $a<0, b \in \mathbb{R}, \mathbf{j}$ denotes the imaginary unit and $\varphi(t-\tau)$ is the outdated state with $\tau>0$ characterising how old the state is. Here (2a) uses only the current state while (2b) uses both the current and outdated states. We assume that $\varphi(t-\tau):=\varphi(t)$ for $0 \leq t<\tau$. We know that system (1) using control (2a) is always stable if $a<0$. Moreover, the convergence speed is determined by $2|a|$. Regarding the stability and the convergence speed for system (1) using control (2b), we have the following result.

Lemma 3.1: If $\tau$ is chosen satisfying $\tau \in\left(0, \tau^{s}\right)$, where $\tau^{s}=\frac{1}{2|b|} \arccos \left(\frac{b^{2}-a^{2}}{a^{2}+b^{2}}\right)$, system (1) using control (2b) is always stable. Assume that $\arg (a+\mathbf{j} b) \in\left(\frac{3}{4} \pi, \pi\right] \bigcup$ $\left(-\pi,-\frac{3}{4} \pi\right)$, where $\arg (\cdot)$ denotes the argument of a complex number. There always exists $\tau^{*}$ such that when $\tau \in\left(0, \tau^{*}\right)$, system (1) using control (2b) has a faster convergence speed than that using control (2a), where $\tau^{*}$ is the minimum positive real number satisfying

$$
\min _{j=1,2}\left\{\tau^{*} \mid \cos \left[\left(b+\delta_{j}\right) \tau^{*}\right]=\frac{\left[a^{2}-b^{2}+b\left(b+\delta_{j}\right)\right] e^{2 a \tau^{*}}}{a^{2}+b^{2}}\right\},
$$

where $\delta_{1}=\sqrt{-a^{2}+e^{-4 a \tau^{*}} a^{2}+e^{-4 a \tau^{*} b^{2}}}$ and $\delta_{2}=-\delta_{1}$.

Proof: For the first statement, note that the closed-loop poles of system (1) using (2b) satisfy

$$
s=\left(1+e^{-s \tau}\right)(a+\mathbf{j} b) .
$$

Letting $s=x+\mathbf{j} y$, it follows that

$$
\begin{aligned}
& x=a\left[1+e^{-\tau x} \cos (y \tau)\right]+b e^{-\tau x} \sin (y \tau), \\
& y=b\left[1+e^{-\tau x} \cos (y \tau)\right]-a e^{-\tau x} \sin (y \tau) .
\end{aligned}
$$

When $\tau=0$, we can obtain $x=2 a$ and $y=2 b$. Because $s$ is continuous with respect to $\tau$, there exists $\tau^{s}$ such that $s$ is always in the open left-half plane when $\tau \in\left(0, \tau^{s}\right)$. To find $\tau^{s}$, let $s=\mathbf{j} \omega_{1}$. Substituting $s=\mathbf{j} \omega_{1}$ into $s=\left(1+e^{-s \tau}\right)(a+\mathbf{j} b)$ gives

$$
\mathbf{j} \omega_{1}=\left(1+e^{-\mathbf{j} \omega_{1} \tau^{s}}\right)(a+\mathbf{j} b),
$$

which implies $-a+\mathbf{j}\left(\omega_{1}-b\right)=e^{-\mathbf{j} \omega_{1} \tau s}(a+\mathbf{j} b)$. After some manipulations, we can obtain $\omega_{1}=2 b$. After separating the real and imaginary parts of (6) and some manipulations, we can obtain $\tau^{s}=\frac{1}{2|b|} \arccos \left(\frac{b^{2}-a^{2}}{a^{2}+b^{2}}\right)$.

For the second statement, note that

$$
\begin{aligned}
\left.\frac{\mathrm{d} x}{\mathrm{~d} \tau}\right|_{\tau=0}= & -\left.a e^{-\tau x}\left(\tau \frac{\mathrm{d} y}{\mathrm{~d} \tau}+y\right) \sin (y \tau)\right|_{\tau=0} \\
& +\left.a e^{-\tau x}\left(-\tau \frac{\mathrm{d} x}{\mathrm{~d} \tau}-x\right) \cos (y \tau)\right|_{\tau=0} \\
& +\left.b e^{-\tau x}\left(\tau \frac{\mathrm{d} y}{\mathrm{~d} \tau}+y\right) \cos (y \tau)\right|_{\tau=0} \\
& +\left.b e^{-\tau x}\left(-\tau \frac{\mathrm{d} x}{\mathrm{~d} \tau}-x\right) \sin (y \tau)\right|_{\tau=0} \\
= & -a x+b y=-2 a^{2}+2 b^{2} .
\end{aligned}
$$

Thus, $\quad \arg (a+\mathbf{j} b) \in\left(\frac{3}{4} \pi, \pi\right] \bigcup\left(-\pi,-\frac{3}{4} \pi\right) \quad$ implies $\left.\frac{\mathrm{d} x}{\mathrm{~d} \tau}\right|_{\tau=0}<0$. Based on the continuity of $\frac{\mathrm{d} x}{\mathrm{~d} \tau}$ with respect to $\tau$, there must exist a neighbourhood $\tau \in\left(0, \tau^{v}\right)$ such that $x$ decreases as $\tau$ increases. Therefore, there exists a positive $\tau^{*}$ satisfying $x\left(\tau^{*}\right)=2 a$ such that $x<2 a$ for $\tau \in\left(0, \tau^{*}\right)$. In order to find $\tau^{*}$, let $s=2 a+\mathbf{j} \omega$. Substituting $s=2 a+\mathbf{j} \omega$ into $s=\left(1+e^{-s \tau}\right)(a+\mathbf{j} b)$ gives

$$
2 a+\mathbf{j} \omega=\left(1+e^{-(2 a+\mathbf{j} \omega) \tau^{*}}\right)(a+\mathbf{j} b),
$$

which implies $a+\mathbf{j}(\omega-b)=e^{-2 a \tau^{*}} e^{-\mathbf{j} \omega \tau^{*}}(a+\mathbf{j} b)$. From (7), we can get that $a^{2}+(\omega-b)^{2}=e^{-4 a \tau^{*}}\left(a^{2}+b^{2}\right)$. It follows that

$$
\omega=b \pm \sqrt{-a^{2}+e^{-4 a \tau^{*}} a^{2}+e^{-4 a \tau^{*} b^{2}} .}
$$

After separating the real and imaginary parts of (7) and after some manipulations, we can obtain

$$
\cos \left(\omega \tau^{*}\right)=\frac{\left(a^{2}+\omega b-b^{2}\right) e^{2 a \tau^{*}}}{a^{2}+b^{2}} .
$$

Substituting (8) into (9), we can see that $\tau^{*}$ is the minimum positive real number satisfying (3), system (1) using control (2b) has a faster convergence speed than that using control (2a) when $\tau \in\left(0, \tau^{*}\right)$.

Remark 1: Lemma 3.1 in Cao and Ren (2009) is a direct result of Lemma 3.1 by noting that the argument of a negative real number is always equal to $\pi$. 


\subsection{Multi-agent consensus with both the current and outdated states under a fixed directed graph}

In this section, we extend the results in Section 3.1 to the multi-agent consensus problem. Consider a multi-agent system with single-integrator kinematics given by

$$
\dot{\varepsilon}_{i}(t)=u_{i}(t), \quad i=1, \ldots, n,
$$

where $\varepsilon_{i}(t)$ and $u_{i}(t)$ denote, respectively, the state and the control input of the $i$-th agent. Consider consensus algorithms using, respectively, only the current states and both the current and outdated states as

$$
\begin{gathered}
u_{i}(t)=-2 \sum_{j=1}^{n} a_{i j}\left[\varepsilon_{i}(t)-\varepsilon_{j}(t)\right], \\
u_{i}(t)=-\sum_{j=1}^{n} a_{i j}\left\{\left[\varepsilon_{i}(t)-\varepsilon_{j}(t)\right]+\left[\varepsilon_{i}(t-\tau)-\varepsilon_{j}(t-\tau)\right]\right\},
\end{gathered}
$$

where $a_{i j}$ is the $(i, j)$-th entry of the adjacency matrix $\mathcal{A}$, and $\varepsilon_{i}(t-\tau), i=1, \ldots, n$ is the outdated state with $\tau>0$ characterising how old the state is. Similar to the discussion in Cao and Ren (2009), we compare (11a) and (11b) in terms of the convergence speed and the maximal control effort. We assume that $\varepsilon_{i}(t-\tau)=\varepsilon_{i}(t), i=1, \ldots, n$ for $0 \leq t<\tau$. System (10) using (11a) can be written in matrix form as

$$
\dot{\xi}(t)=-2 \mathcal{L} \xi(t),
$$

where $\xi=\left[\varepsilon_{1}, \varepsilon_{2}, \ldots, \varepsilon_{n}\right]^{T}$ and $\mathcal{L}$ is the Laplacian matrix defined in Section 2. Let $\lambda_{i}=a_{i}+\mathbf{j} b_{i}$ be the $i$-th eigenvalue of $\mathcal{L}$, where $a_{i}, b_{i} \in \mathbb{R}$. Suppose that the directed communication graph has a directed spanning tree. Without loss of generality, we label $\lambda_{i}$ by the magnitudes of the real parts as $0=a_{1}<a_{2} \leq \cdots \leq a_{n}$. We know that system (10) using control (11a) achieves consensus with a convergence speed $2\left|a_{2}\right|$. We have the following result for (10) using (11b).

Theorem 3.2: If $\tau$ is chosen satisfying $\tau \in\left(0, \tau^{s}\right)$, where $\tau^{s}=\min _{i=2, \ldots, n}\left\{\tau_{i}^{s}\right\}$ and $\tau_{i}^{s}=\frac{1}{2\left|b_{i}\right|} \operatorname{arc} \cos \left(\frac{b_{i}^{2}-a_{i}^{2}}{a_{i}^{2}+b_{i}^{2}}\right)$, system (10) using control (11b) achieves consensus. Assume that $\arg \left(\lambda_{i}\right) \in\left(-\frac{1}{4} \pi, \frac{1}{4} \pi\right), \forall i=2,3, \ldots, n$, holds. There always exists $\tau^{*}$ such that when $\tau \in\left(0, \tau^{*}\right)$, system (10) using (11b) achieves consensus with a faster convergence speed than that using (11a), where $\tau^{*}=\min _{i=2, \ldots, n}\left\{\tau_{i}\right\}$, and $\tau_{i}$ is the minimal positive number satisfying

$\min _{j=1,2}\left\{\tau_{i} \mid \cos \left[\left(-b_{i}+\delta_{i j}\right) \tau_{i}\right]=\frac{\left[a_{i}^{2}-b_{i}^{2}-b_{i}\left(-b_{i}+\delta_{i j}\right)\right] e^{-2 a_{i} \tau_{i}}}{a_{i}^{2}+b_{i}^{2}}\right\}$,

where $\delta_{i 1}=\sqrt{-a_{i}^{2}+e^{4 a_{i} \tau_{i}} a_{i}^{2}+e^{4 a_{i} \tau_{i}} b_{i}^{2}}$ and $\delta_{i 2}=-\delta_{i 1}$. In addition, the maximum control effort using (11b) is equal to that using (11a). In particular, we can get that $\max _{i} \max _{t \geq 0}\left|u_{i}(t)\right|=\max _{i}\left|u_{i}(0)\right|$.

Proof: For the first statement, note that system (10) using (11b) can be written in matrix form as

$$
\dot{\xi}(t)=-\mathcal{L} \xi(t)-\mathcal{L} \xi(t-\tau) .
$$

By applying the Laplace transform to both sides of (13), it follows that

$$
\left(s I_{n}+\mathcal{L}+e^{-s \tau} \mathcal{L}\right) \xi(s)=\xi(0) .
$$

Thus the closed-loop poles of system (10) using (11b) satisfy

$$
\operatorname{det}\left[\left(s I_{n}+\left(1+e^{-s \tau}\right) \mathcal{L}\right]=0 .\right.
$$

Noting that $\lambda_{i}$ is the $i$-th eigenvalue of $\mathcal{L}$, it follows that the closed-loop poles of system (10) using (11b) satisfy

$$
s+\left(1+e^{-s \tau}\right) \lambda_{i}=0, \quad i=1,2, \ldots, n .
$$

Because $\lambda_{1}=0$, it follows from (14) that $s=0$. Because $-a_{i}<0, i=2, \ldots, n$, by letting $-\lambda_{i}$ play the role of $a+\mathbf{j} b$ in (4), it follows that the closed-loop poles of (14) corresponding to $-\lambda_{i}, i=2, \ldots, n$, are always in the open left-half plane if $\tau \in\left(0, \tau_{j}^{S}\right), i=2, \ldots, n$. Noting that the eigenvector associated with $s=0$ is $\mathbf{1}_{n}$, it then follows that (10) using control (11b) achieves consensus if $\tau$ is chosen satisfying $\tau \in\left(0, \tau^{s}\right)$.

For the second statement, if $\tau \in\left(0, \tau^{*}\right)$, where $\tau^{*} \leq \tau_{i}, \quad i=2, \ldots, n$, the closed-loop poles of (14) corresponding to $-\lambda_{i}, i=2, \ldots, n$ are always on the left-hand side of $-\lambda_{i}$. It follows that if $\tau \in\left(0, \tau^{*}\right)$ system (10) using control (11b) achieve consensus with a faster convergence speed than that using (11a).

For the third statement, the proof of the maximum control effort is similar to that of Lemma 3.4 in Cao and Ren (2009) by noting that $e^{-2 \mathcal{L} t}$ is also a (row) stochastic matrix if $\mathcal{L}$ is associated with a directed graph (Ren and Beard 2005).

Remark 2: Note that the distribution of the eigenvalues of a nonsymmetric Laplacian matrix associated with a directed graph is related to the number of agents in the directed graph (Agaev and Chebotarev 2005). In particular, $-\left(\frac{\pi}{2}-\frac{\pi}{n}\right) \leq \arg \left(\lambda_{i}\right) \leq \frac{\pi}{2}-\frac{\pi}{n}, i=1,2 \ldots, n$. If the number of agents in the communication graph is less than $4, \arg \left(\lambda_{i}\right) \in\left(-\frac{1}{4} \pi, \frac{1}{4} \pi\right)$ is satisfied. However, if the number of agents is equal to or more than 4 , we should first check the eigenvalues of the corresponding Laplacian matrix to ensure that they satisfy $\arg \left(\lambda_{i}\right) \in\left(-\frac{1}{4} \pi, \frac{1}{4} \pi\right)$. In contrast to the directed graph case, all eigenvalues of the Laplacian matrix associated with an undirected graph are real numbers, thus satisfying $\arg \left(\lambda_{i}\right) \in\left(-\frac{1}{4} \pi, \frac{1}{4} \pi\right)$. Therefore, when the fixed graph is undirected, there always exists $\tau^{*}$ such that for arbitrary number of agents, system (10) using 
control (11b) can achieve consensus with a faster convergence speed than that using (11a) when $\tau \in\left(0, \tau^{*}\right)$.

Remark 3 : The proposed consensus algorithm using both the current and outdated states can achieve a faster convergence speed than the standard one without increasing the maximal control effort. The tradeoff is that each agent needs more memory room to store the outdated states from its neighbours over a past period of time. The time period is generally small and adjustable. In real applications, it is sufficient to store the outdated states at a certain frequency over a small period of time. Because memory chips become cheaper and more cost-efficient, the proposed consensus algorithm using both the current and outdated states provides an option to applications where the convergence speed plays a more important role than the available memory room.

Remark 4: Note that here the bound for $\tau$ depends on the global information, that is, the eigenvalues of the Laplacian matrix. In this article, we assume that the bound can be computed offline before running the algorithm. In fact, it is quite common that the bounds of the control parameters depend on the eigenvalues of the Laplacian matrix in the current literature on distributed control (see e.g. Ren, Beard, and Atkins 2007; Ren and Atkins 2007). However, the distributed nature of the algorithm lies in the fact that each agent only interacts with its neighbours to reach consensus. How to obtain the eigenvalues of the Laplacian matrix (and hence the bound for $\tau$ ) in a distributed manner is still an open challenging question that deserves further investigation.

\subsection{Multi-agent consensus with both the current and outdated states under dynamic directed graphs}

In this section, we extend the result in Section 3.2 to the case of dynamic interaction. In this case, (11a) and (11b) can be rewritten as

$$
\begin{gathered}
u_{i}(t)=-2 \sum_{j=1}^{n} a_{i j}(t)\left[\varepsilon_{i}(t)-\varepsilon_{j}(t)\right] \\
u_{i}(t)=-\sum_{j=1}^{n} a_{i j}(t)\left\{\left[\varepsilon_{i}(t)-\varepsilon_{j}(t)\right]+\left[\varepsilon_{i}(t-\tau)-\varepsilon_{j}(t-\tau)\right]\right\},
\end{gathered}
$$

where $a_{i j}(t)$ is time-varying. Here, we apply the dwell time to the continuous-time algorithms (11a) and (11b), which means that the communication graphs are constrained to switch only at discrete-time instants, i.e. $\mathcal{L}(t)$ is piecewise constant. Assume that the communication graph remains constant for $t \in\left[\eta_{k}, \eta_{k+1}\right), k=0,1, \ldots$ and switches at $t=\eta_{k}, k=$ $0,1, \ldots$, where $\eta_{k}>\eta_{k-1}$. Here, we also assume that $\eta_{k+1}-\eta_{k}>\tau, k=0,1, \ldots$ Define $\varepsilon_{i}(t-\tau)=\varepsilon_{i}(t)$ for $t \in\left[\eta_{k}, \eta_{k}+\tau\right)$. It has been shown in Ren and Beard (2005) that if the directed graph has a directed spanning tree at every time interval, system (10) using (15a) can achieve consensus. For system (10) using (15b), we have the following result.

Theorem 3.3: Let $\mathcal{G}[k] \in \overline{\mathcal{G}}$ be a switching communication graph at time $t=\eta_{k}, k=0,1, \ldots$, where $\overline{\mathcal{G}}$ denotes the set of directed graphs that have a directed spanning tree. Assume that $\arg \left(\lambda_{i}(\mathcal{G}[k])\right) \in\left(-\frac{1}{4} \pi, \frac{1}{4} \pi\right), \quad i=$ $2,3, \ldots, n$, holds, $\forall \mathcal{G}[k] \in \overline{\mathcal{G}}$. If $\tau$ satisfies $\tau \in\left(0, \tau^{*}\right)$, where $\tau^{*}=\min _{\overline{\mathcal{G}}} \min _{i=2, \ldots, n}\left\{\tau_{i}\right\}$ and $\tau_{i}$ is defined in Theorem 3.2. Then system (10) using (15b) can achieve consensus with a faster convergence speed than that using (15a).

Proof: We define a positive-definite discrete Lyapunov function candidate $\quad V\left(\xi\left(\eta_{k}\right)\right)=\max _{i}\left\{\varepsilon_{i}\left(\eta_{k}\right)\right\}-$ $\min _{i}\left\{\varepsilon_{i}\left(\eta_{k}\right)\right\}$ with respect to the desired equilibrium set $\left\{\varepsilon_{1}=\cdots=\varepsilon_{n}\right\}$, where $\xi(t)$ was defined in (12). We next show that $V\left(\xi\left(\eta_{k+1}\right)\right)<V\left(\xi\left(\eta_{k}\right)\right), \forall k=0,1, \ldots$

Considering the time interval $t \in\left[\eta_{k}, \eta_{k+1}\right)$, we separate this time interval into two parts $\left[\eta_{k}, \eta_{k}+\tau\right) \bigcup\left[\eta_{k}+\tau, \eta_{k+1}\right)$. For $t \in\left[\eta_{k}, \eta_{k}+\tau\right)$, we define a continuous function $V^{\prime}(\xi(t))=\max _{i}\left\{\varepsilon_{i}(t)\right\}-$ $\min _{i}\left\{\varepsilon_{i}(t)\right\}$. Because system (10) using (15b) is the same as that using (15a) for $t \in\left[\eta_{k}, \eta_{k}+\tau\right)$, we can obtain that $V^{\prime}\left(\xi\left(\eta_{k}\right)\right)>\lim _{t \rightarrow \eta_{k}+\tau} V^{\prime}(\xi(t))=V^{\prime}\left(\xi\left(\eta_{k}+\tau\right)\right)$ using a similar analysis to that in Moreau (2004a) by noting that the directed graph $\mathcal{G}$ always has a directed spanning tree for $t \in\left[\eta_{k}, \eta_{k}+\tau\right)$. Then by following a similar analysis to that in the proof of Lemma 3.4 in Cao and Ren (2009), it is easy to verify that $\|\xi(t)\|_{\infty} \leq$ $\left\|\xi\left(\eta_{k}+\tau\right)\right\|_{\infty}$ for $t \in\left[\eta_{k}+\tau, \eta_{k+1}\right)$, which implies that

$$
\max _{i}\left\{\varepsilon_{i}\left(\eta_{k+1}\right)\right\}=\lim _{t \rightarrow \eta_{k+1}} \max _{i}\left\{\varepsilon_{i}(t)\right\} \leq \max _{i}\left\{\varepsilon_{i}\left(\eta_{k}+\tau\right)\right\} .
$$

Similarly, we can also get that

$$
\min _{i}\left\{\varepsilon_{i}\left(\eta_{k+1}\right)\right\}=\lim _{t \rightarrow \eta_{k+1}} \min _{i}\left\{\varepsilon_{i}(t)\right\} \geq \min _{i}\left\{\varepsilon_{i}\left(\eta_{k}+\tau\right)\right\} .
$$

Thus, it follows that $V^{\prime}\left(\xi\left(\eta_{k}+\tau\right)\right) \geq V^{\prime}\left(\xi\left(\eta_{k+1}\right)\right)$. Combining the previous arguments shows that $V^{\prime}\left(\xi\left(\eta_{k}\right)\right)>V^{\prime}\left(\xi\left(\eta_{k+1}\right)\right)$. Noting that $V\left(\xi\left(\eta_{k}\right)\right)=V^{\prime}\left(\xi\left(\eta_{k}\right)\right)$ and $V\left(\xi\left(\eta_{k+1}\right)\right)=V^{\prime}\left(\xi\left(\eta_{k+1}\right)\right)$, it follows that $V\left(\xi\left(\eta_{k}\right)\right)>V\left(\xi\left(\eta_{k+1}\right)\right), \quad \forall k=0,1, \ldots \quad$ Therefore, it follows that $V\left(\xi\left(\eta_{k}\right)\right) \rightarrow 0$ as $k \rightarrow \infty$, which means that consensus is achieved.

For the convergence speed, when $t \in\left[\eta_{k}, \eta_{k+1}\right)$, $k=0,1, \ldots$, by following a similar analysis to that in Theorem 3.2, the convergence speed using (15b) is faster than that using (15a). Thus, based on the continuity of the state along time, system (10) using (15b) 
can achieve consensus with a faster convergence speed than that using (15a).

\section{Discrete-time case}

In this section, we study a discrete-time consensus algorithm with both the current and outdated states. We first discuss the stability and convergence condition in the single-agent case and then extend the single-agent case to the multi-agent case.

\subsection{Single-agent case}

The discrete-time case of (1) can be written as

$$
\frac{\varnothing(k+1)-\varnothing(k)}{T}=u(k),
$$

where $k$ is the discrete-time index, $T$ is the sampling period, $\varnothing(k)=\varnothing(k T)$, and $u(k)=u(k T)$. We study two different algorithms using, respectively, only the current state and both the current and outdated states as

$$
\begin{gathered}
u(k)=-2 \alpha \varnothing(k) \\
u(k)=-\alpha \varnothing(k)-\alpha \varnothing(k-N),
\end{gathered}
$$

where $\varnothing(k-N)$ is the outdated state with $N>0$ characterising how old the state is. We assume that $\varnothing(k-N):=\varnothing(k)$ when $0 \leq k<N$. Using (17a) for system (16), we can get that $\varnothing(k+1)=(1-2 \alpha T) \varnothing(k)$. When $|1-2 \alpha T|<1$, i.e. $0<\alpha T<1$, system (16) using (17a) is stable. Moveover, the convergence speed is determined by $|1-2 \alpha T|$. For system (16) using (17b), we have the following result.

Lemma 4.1: When $0<\alpha T<1$, system (16) using (17b) is always stable, $\forall N>0$.

Proof: Using (17b), (16) can be written in matrix form as

$$
\Phi(k+1)=P \Phi(k),
$$

where $\Phi(k)=[\varnothing(k), \ldots, \varnothing(k-N)]^{T} \in \mathbb{R}^{N+1}$, and

$$
P=\left[\begin{array}{cccc}
1-\alpha T & 0 & \cdots & -\alpha T \\
1 & 0 & \cdots & 0 \\
0 & \ddots & 0 & 0 \\
\vdots & 0 & 1 & 0
\end{array}\right] .
$$

We next show that all eigenvalues of $P$ are within the unit circle if $0<\alpha T<1$. Note that the characteristic polynomial of $P$ is given by $\operatorname{det}\left(\mu I_{N+1}-P\right)=0$, which is equal to

$$
\mu^{N+1}-(1-\alpha T) \mu^{N}+\alpha T=0 .
$$

Based on the Gershgorin disc theorem (Horn and Johnson 1985), if $0<\alpha T<1$, the eigenvalues $\mu$ of $P$ must be within or on the unit circle. We next prove that the eigenvalues of $P$ cannot be on the unit circle by contradiction. Substituting $\mu=e^{\mathbf{j} \theta}$ into characteristic polynomial (19), we get that

$$
e^{(N+1) \mathbf{j} \theta}-(1-\alpha T) e^{N \mathbf{j} \theta}+\alpha T=0 .
$$

By separating the real and imaginary parts of (20), we can obtain

$$
\begin{gathered}
\cos [(N+1) \theta]-(1-\alpha T) \cos (N \theta)+\alpha T=0, \\
\sin [(N+1) \theta]-(1-\alpha T) \sin (N \theta)=0 .
\end{gathered}
$$

By combining (21a) and (21b), after some manipulations, we can obtain $\cos (N \theta)=-1$, which implies $N \theta=(2 p+1) \pi, p \in \mathbb{Z}$, where $\mathbb{Z}$ denotes the integer set. Substituting $\cos (N \theta)=-1$ into (21a) gives that $\cos (N+1) \theta=-1$, which implies that $(N+1) \theta=$ $(2 q+1) \pi, q \in \mathbb{Z}$. Then we can get that $\theta=2(p-q) \pi$, which implies that $\cos (N \theta)=0$. This contradicts the fact that $\cos (N \theta)=-1$. Thus all eigenvalues of $P$ are within the unit circle, which implies that system (16) using (17b) is stable if $0<\alpha T<1$.

Lemma 4.2: For any positive integer number $N$, there always exists $\sigma$ such that when $\alpha T \in(0, \sigma)$, system (16) using (17b) has a faster convergence speed than that using (17a), where $\sigma$ is the minimum positive number satisfying

$$
(1-2 \sigma)^{N+1} \sin (N+1) \theta=(1-\sigma)(1-2 \sigma)^{N} \sin (N \theta),
$$

where $\theta$ is given by

$$
\theta=\frac{1}{N} \arccos \left[\frac{(1-2 \sigma)^{2 N}\left(3 \sigma^{2}-2 \sigma\right)-\sigma^{2}}{2 \sigma(1-\sigma)(1-2 \sigma)^{N}}\right] .
$$

Proof: First, we show the existence of $\sigma$ for any $N$. Denote $\rho:=1-2 \alpha T$ as the eigenvalue of system (16) using (17a). We can obtain that $\left.\rho\right|_{\alpha T=0}=1$ and $\left.\frac{\mathrm{d} \rho}{\mathrm{d}(\alpha T)}\right|_{\alpha T=0}=-2$. When $\alpha T=0$, system (16) using (17b) has exactly one eigenvalue equal to 1 and $N$ zero eigenvalues. Then differentiating (19) with respect to $\alpha T$ gives

$$
(N+1) \mu^{N} \frac{\mathrm{d} \mu}{\mathrm{d}(\alpha T)}-(1-\alpha T) N \mu^{N-1} \frac{\mathrm{d} \mu}{\mathrm{d}(\alpha T)}+\mu^{N}+1=0 .
$$

It follows that $\left.\frac{\mathrm{d} \mu}{\mathrm{d}(\alpha T)}\right|_{\alpha T=0, \mu=1}=-2$, which is equal to

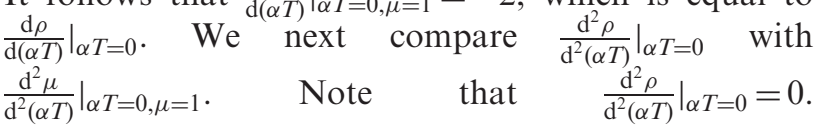


Differentiating (24) with respect to $\alpha T$ gives that

$$
\begin{aligned}
& (N+1) N \mu^{N-1}\left(\frac{\mathrm{d} \mu}{\mathrm{d}(\alpha T)}\right)^{2}+(N+1) \mu^{N} \frac{\mathrm{d}^{2} \mu}{\mathrm{d}^{2}(\alpha T)}+N \mu^{N-1} \\
& \times \frac{\mathrm{d} \mu}{\mathrm{d}(\alpha T)}-(1-\alpha T) N(N-1) \mu^{N-2}\left(\frac{\mathrm{d} \mu}{\mathrm{d}(\alpha T)}\right)^{2} \\
& -(1-\alpha T) N \mu^{N-1} \frac{\mathrm{d}^{2} \mu}{\mathrm{d}^{2}(\alpha T)}+N \mu^{N-1} \frac{\mathrm{d} \mu}{\mathrm{d}(\alpha T)}=0
\end{aligned}
$$

It follows that $\left.\frac{\mathrm{d}^{2} \mu}{\mathrm{d}^{2}(\alpha T)}\right|_{\alpha T=0, \mu=1}=-4 N<0$, which implies that $\left.\frac{\mathrm{d}^{2} \mu}{\mathrm{d}^{2}(\alpha T)}\right|_{\alpha T=0, \mu=1}<\left.\frac{\mathrm{d}^{2} \rho}{\mathrm{d}^{2}(\alpha T)}\right|_{\alpha T=0}$. Based on the continuity of $\rho$ and $\mu$ with respect to $\alpha T$, there always exists a positive $\sigma$ such that when $\alpha T \in(0, \sigma)$, all eigenvalues of $P$ have modules smaller than $|1-2 \alpha T|$, which implies that system (16) using (17b) has a faster convergence speed than that using (17a).

Next, we show how to obtain $\sigma$. We know that as $\alpha T$ increases from 0 continuously, $\sigma$ can be obtained when $|\mu|$ first approaches $(1-2 \alpha T)$. Letting $\mu=(1-2 \sigma) e^{\mathbf{j} \theta}$, it follows that (19) can be written as the following two equations when considering the real and imaginary parts separately:

$$
\begin{gathered}
(1-2 \sigma)^{N+1} \cos (N+1) \theta-(1-\sigma)(1-2 \sigma)^{N} \\
\times \cos (N \theta)+\sigma=0, \\
(1-2 \sigma)^{N+1} \sin (N+1) \theta-(1-\sigma)(1-2 \sigma)^{N} \sin (N \theta)=0 .
\end{gathered}
$$

Combining the above two equations, after some manipulations, we can obtain (23). Thus $\sigma$ is the minimum positive number satisfying (22), where $\theta$ is given in (23).

Remark 5: If we let $N=1$, it is easy to verify that system (16) using (17b) has a faster convergence than that using (17a) if $0<\alpha T<\frac{1}{4}$.

\subsection{Multi-agent case}

In this section, we extend the single-agent case in Section 4.1 to the multi-agent case. For a multi-agent system with discrete-time single-integrator kinematics given by

$$
\frac{\varsigma_{i}(k+1)-S_{i}(k)}{T}=u_{i}(k), \quad i=1,2, \ldots, n,
$$

where $k$ is the discrete-time index, $T$ is the sampling period, $\varsigma_{i}(k)=\varsigma_{i}(k T)$ and $u_{i}(k)=u_{i}(k T)$, we study the consensus algorithms using, respectively, only the current states and both the current and outdated states as

$$
\begin{aligned}
& u_{i}(k)=-2 \sum_{j=1}^{n} a_{i j}\left[\varsigma_{i}(k)-\varsigma_{j}(k)\right], \\
u_{i}(k)= & -\sum_{j=1}^{n} a_{i j}\left[\varsigma_{i}(k)-\varsigma_{j}(k)\right] \\
& -\sum_{j=1}^{n} a_{i j}\left[\varsigma_{i}(k-N)-\varsigma_{j}(k-N)\right],
\end{aligned}
$$

where $a_{i j}$ is the $(i, j)$-th entry of the adjacency matrix $\mathcal{A}$ associated with an undirected graph $\mathcal{G}$, and $\varsigma_{i}(k-N)$ is the outdated state with $N>0$ characterising how old the state is. We here assume that $\varsigma_{i}(k-N):=\varsigma_{i}(k)$ when $0 \leq k<N$. We rewrite system (26) using (27a) and system (26) using (27b) in matrix forms as

$$
\begin{gathered}
\chi(k+1)=\left(I_{n}-2 T \mathcal{L}\right) \chi(k), \\
\chi(k+1)=\left(I_{n}-T \mathcal{L}\right) \chi(k)-T \mathcal{L} \chi(k-N),
\end{gathered}
$$

where $\chi(k)=\left[\varsigma_{1}(k), \varsigma_{2}(k), \ldots, \varsigma_{n}(k)\right]^{T}, I_{n}$ denotes the $n \times n$ identity matrix, and $\mathcal{L}$ is the Laplacian matrix associated with $\mathcal{A}$.

Let $\lambda_{i}, i=1,2, \ldots n$, be the eigenvalues of $\mathcal{L}$. When the undirected graph $\mathcal{G}$ is connected, we know that $\mathcal{L}$ has a simple zero eigenvalue and all other eigenvalues are positive (Agaev and Chebotarev 2000). Without loss of generality, we label $\lambda_{i}$ as $0=\lambda_{1}<\lambda_{2} \leq \cdots \leq \lambda_{n}$. Let $\rho_{i}$ denote the $i$-th eigenvalue of $(I-2 T \mathcal{L})$. Note that $\rho_{i}=1-2 T \lambda_{i}$. When $\lambda_{1}=0, \rho_{1}=1$. Also note $(I-2 T \mathcal{L}) \mathbf{1}_{n}=\mathbf{1}_{n}$ from the fact that $\mathcal{L} \mathbf{1}_{n}=0$. When $T<\frac{1}{\lambda_{n}}$, it follows that $\left|\rho_{i}\right|<1, i=2, \ldots, n$. Therefore, (28a) achieves consensus if $T<\frac{1}{\lambda_{n}}$.

Note that (28b) can be rewritten as

$$
\Gamma(k+1)=F \Gamma(k),
$$

where $\Gamma(k)=[\chi(k), \chi(k), \ldots, \chi(k-N)] \in \mathbb{R}^{n(N+1) \times 1}$ and $F \in \mathbb{R}^{n(N+1) \times n(N+1)}$ is given by

$$
F=\left(\begin{array}{ccccc}
I_{n}-T \mathcal{L} & 0 & \ldots & 0 & -T \mathcal{L} \\
I_{n} & 0 & \ldots & 0 & 0 \\
0 & I_{n} & 0 & \ldots & 0 \\
\vdots & 0 & \ddots & 0 & 0 \\
0 & 0 & 0 & I_{n} & 0
\end{array}\right) .
$$

We next study the stability and the convergence speed of system (26) using (27b).

Theorem 4.3: For any positive integer $N$, system (26) using (27b) achieves consensus if $T<\frac{1}{\lambda_{n}}$. Moreover, there always exists $T^{*}$ such that when $T \in\left(0, T^{*}\right)$, system (26) using (27b) has a faster convergence speed than that using (27a), where $T^{*}=\min _{i=2,3, \ldots, n}\left\{T_{i}\right\}$ and $T_{i}$ is the 
minimum positive number satisfying (22) with $\lambda_{i} T_{i}$ replacing $\sigma$ in (22) and (23). The maximum control effort using (27b) is equal to that using (27a). In particular, we can get that $\max _{i} \max _{k \geq 0}\left|u_{i}(k)\right|=$ $\max _{i}\left|u_{i}(0)\right|$.

Proof: Because $\mathcal{L}$ is a symmetrical matrix, it follows that $\mathcal{L}$ can be written as $\mathcal{L}:=M^{T} \Lambda M$, where $\Lambda=\operatorname{diag}\left(\lambda_{1}, \ldots, \lambda_{n}\right)$. Then $F$ can be transformed to $F^{\prime}$, where $F^{\prime}$ becomes

$$
F^{\prime}=\mathbb{M} F \mathbb{M}^{T}=\left(\begin{array}{ccccc}
I_{n}-T \Lambda & 0 & \ldots & 0 & -T \Lambda \\
I_{n} & 0 & \ldots & 0 & 0 \\
0 & I_{n} & 0 & \ldots & 0 \\
\vdots & 0 & \ddots & 0 & 0 \\
0 & 0 & 0 & I_{n} & 0
\end{array}\right),
$$

where

$$
\mathbb{M}=\left(\begin{array}{cccc}
M & 0 & \ldots & 0 \\
0 & M & \ldots & 0 \\
\vdots & 0 & \ddots & \vdots \\
0 & \ldots & 0 & M
\end{array}\right)
$$

Note that $F^{\prime}$ has a similar structure to $P$ in (18) and the eigenvalues of $F$ are the same as those of $F^{\prime}$. By mimicking a similar analysis to that of (18), it follows that

$$
\begin{aligned}
\operatorname{det}\left(s I_{(N+1) n}-F^{\prime}\right)= & \operatorname{det}\left(\left[S I_{n}-\left(I_{n}-T \Lambda\right)\right] s^{N}\right. \\
& \left.+(-1)^{N+2}(-1)^{N} T \Lambda\right) \\
= & \operatorname{det}\left(s^{N+1} I_{n}-\left(I_{n}-T \Lambda\right) s^{N}+T \Lambda\right)=0 .
\end{aligned}
$$

Thus the eigenvalues of $F$, denoted by $\mu_{i}$, satisfy $\mu_{i}^{N+1}-\left(1-T \lambda_{i}\right) \mu_{i}^{N}+T \lambda_{i}=0$. Note that each eigenvalue of $\mathcal{L}, \lambda_{i}$, corresponds to $N+1$ eigenvalues of $F$, denoted as $\mu_{i,}, j=1,2, \ldots, N+1$, for $i=1,2, \ldots, n$.

When $\lambda_{1}=0, \mu_{1_{1}}=1$ and $\mu_{1_{j}}=0, j=2, \ldots, N+1$. Therefore, $F$ has one eigenvalue equal to one with a corresponding eigenvector $\mathbf{1}_{n(N+1)}$. Based on the analysis in Lemma 4.1, if $0<\lambda_{i} T<1, i=2, \ldots, n$, all eigenvalues $\left|\mu_{i_{i}}\right|<1, j=1, \ldots, N+1$. Therefore, system (29) achieves consensus if $T<\frac{1}{\lambda_{n}}$.

For the convergence speed, by replacing $\sigma$ with $\lambda_{i} T_{i}, \quad i=2,3, \ldots, n$ in Lemma 4.2 , when $T^{*} \leq T_{i}$, $i=2, \ldots, n$, it follows that $\left|\mu_{i_{i}}\right|<\left|1-2 T \lambda_{i}\right|, \quad \forall j=$ $1,2, \ldots, N+1$, for each $i=2,3, \ldots, n$, where $1-2 T \lambda_{i}$, $i=2, \ldots, n$, are the corresponding eigenvalues of (28a). Therefore, it follows that system (26) using (27b) can achieve a faster convergence speed than that using (27a) when $T \in\left(0, T^{*}\right)$.

For the maximum control effort, we rewrite (27a) in matrix form as $U(k)=-2 \mathcal{L} \chi(k)$ and $U(0)=$ $-2 \mathcal{L} \chi(0)$, where $U(k)=\left[u_{1}(k), \ldots, u_{n}(k)\right] \in \mathbb{R}^{n} . \quad$ It follows from (28a) that $\chi(k)=(I-2 T \mathcal{L})^{k} \chi(0)$.
Therefore, we can obtain that $U(k)=(I-2 T \mathcal{L})^{k} U(0)$ from the fact that $\mathcal{L}(I-2 T \mathcal{L})=(I-2 T \mathcal{L}) \mathcal{L}$. It then follows that $\|U(k)\|_{\infty} \leq\left\|(I-2 T \mathcal{L})^{k}\right\|_{\infty}\|U(0)\|_{\infty}=$ $\|U(0)\|_{\infty}$ by noting that $(I-2 T \mathcal{L})^{k}$ is a (row) stochastic matrix. Thus the maximum control effort using (27a) is $\max _{i}\left|u_{i}(0)\right|$. For $(28 \mathrm{~b})$, when $0 \leq k<N,(27 \mathrm{~b})$ is identical to (27a). Thus we know that $\|U(k)\|_{\infty} \leq\|U(0)\|_{\infty}$ for $0 \leq k<N . \quad$ When $\quad k=N, \quad\|U(k)\|_{\infty}=\|-\mathcal{L} \chi(0)-$ $\mathcal{L} \chi(N)\left\|_{\infty}=\right\|-\mathcal{L} \chi(0)-\mathcal{L}(I-2 T \mathcal{L})^{N} \chi(0)\left\|_{\infty} \leq\right\| I+(I-$ $2 T L)^{N}\left\|_{\infty}\right\| \mathcal{L} \chi(0)\left\|_{\infty} \leq\right\| U(0) \|_{\infty}$. When $k>N$, by noting that (28b) achieves consensus, we can obtain that $\|U(k)\|_{\infty} \leq\|U(N)\|_{\infty}$ for $k>N$ by following a similar analysis to that in the proof of Lemma 3.4 in Cao and Ren (2009). Therefore, we know that $\|U(k)\|_{\infty} \leq$ $\|U(0)\|_{\infty}$ for $k>N$. In summary, we have that $\|U(k)\|_{\infty} \leq\|U(0)\|_{\infty}$ for $k \geq 0$ and the maximal control effort using (27b) is $\max _{i}\left|u_{i}(0)\right|$. Combining the previous arguments shows that the maximum control effort using (27b) is equal to that using (27a). In particular, we get that $\max _{i} \max _{k \geq 0}\left|u_{i}(k)\right|=\max _{i}\left|u_{i}(0)\right|$.

Remark 6: Similar to the continuous-time case, the proposed discrete-time consensus algorithm using both the current and outdated states requires more memory room to store the outdated states.

Remark 7: Note that the algorithm proposed in this section actually extends the accelerated algorithm proposed in Ghosh et al. (1998) from the case where the time-delayed information must be exactly the last sample before the current one to the case where the time-delayed information can be arbitrarily outdated.

\section{Simulation}

In this section, we compare the consensus algorithms using both the current and outdated states with the standard ones in both the continuous-time and discrete-time settings. For control (11a) and control (11b), we choose $\tau=0.2 \mathrm{~s}$ and

$$
\mathcal{L}=\left(\begin{array}{cccccc}
2 & -1 & -1 & 0 & 0 & 0 \\
-1 & 1 & 0 & 0 & 0 & 0 \\
-1 & 0 & 2 & -1 & 0 & 0 \\
0 & 0 & -1 & 1 & 0 & 0 \\
0 & -1 & -1 & -1 & 3 & 0 \\
0 & 0 & 0 & 0 & -1 & 1
\end{array}\right) .
$$

Figures 1 and 2 show the convergence results and control efforts of system (10) using, respectively, (11a) and (11b) while Table 1 shows the convergence time using (11a) and (11b). We can see that the convergence speed of multi-agent consensus with both the current and outdated states is improved if $\tau$ is chosen properly under a directed fixed communication graph. Also, the 


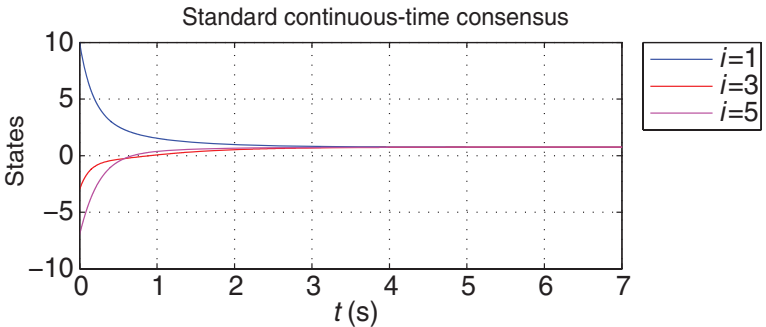

Continuous-time consensus with both the current and outdated states

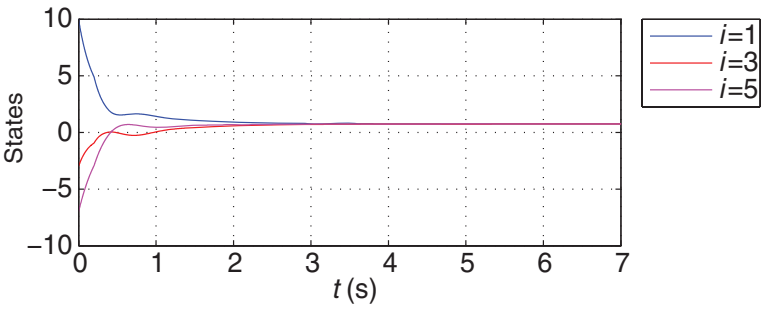

Figure 1. Convergence results using (11a) and (11b) under fixed directed interaction.
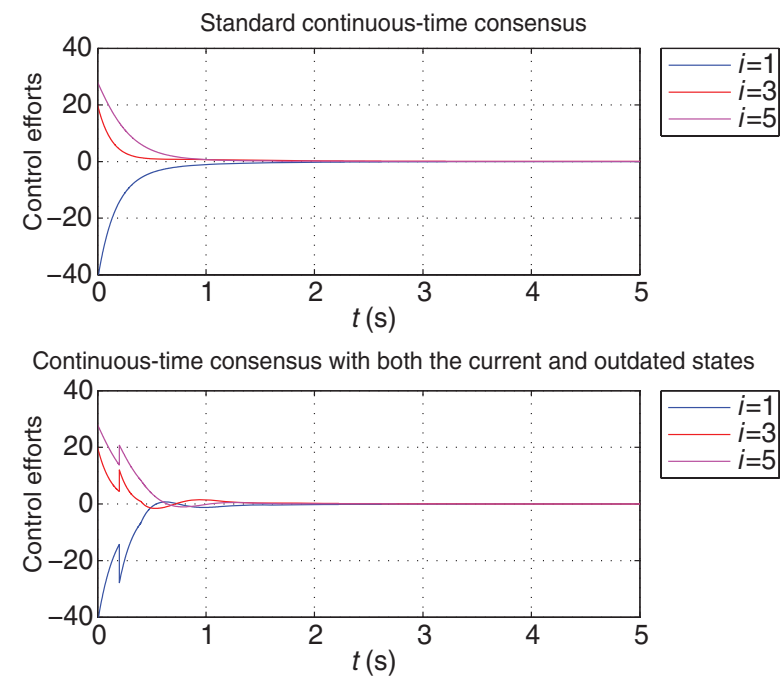

Figure 2. Control efforts using (11a) and (11b) under fixed directed interaction.

maximum control effort for the consensus algorithm using both the current and outdated states is identical to that for the standard one.

In the case of dynamic directed interaction, we choose $\tau=0.2 \mathrm{~s}$. We assume that $\mathcal{L}(t)$ switches among $\left\{\mathcal{L}_{1}, \mathcal{L}_{2}, \mathcal{L}_{3}, \mathcal{L}_{4}\right\}$, where

$$
\mathcal{L}_{1}=\left(\begin{array}{cccccc}
2 & -1 & -1 & 0 & 0 & 0 \\
-1 & 1 & 0 & 0 & 0 & 0 \\
-1 & 0 & 2 & -1 & 0 & 0 \\
0 & 0 & -1 & 1 & 0 & 0 \\
0 & -1 & -1 & -1 & 3 & 0 \\
0 & 0 & 0 & 0 & -1 & 1
\end{array}\right),
$$

Table 1. Convergence time comparison in the continuoustime case under fixed directed interaction.

\begin{tabular}{lcc}
\hline & $\begin{array}{c}\text { Within 5\% } \\
\text { of final } \\
\text { equilibrium }\end{array}$ & $\begin{array}{c}\text { Within 2\% } \\
\text { of final } \\
\text { equilibrium }\end{array}$ \\
\hline System (10) using (11a) & $4.42 \mathrm{~s}$ & $5.07 \mathrm{~s}$ \\
System (10) using (11b) & $3.74 \mathrm{~s}$ & $\begin{array}{c}4.29 \mathrm{~s} \\
\text { Convergence time }\end{array}$ \\
\begin{tabular}{l} 
improvement percentage \\
\hline
\end{tabular}
\end{tabular}

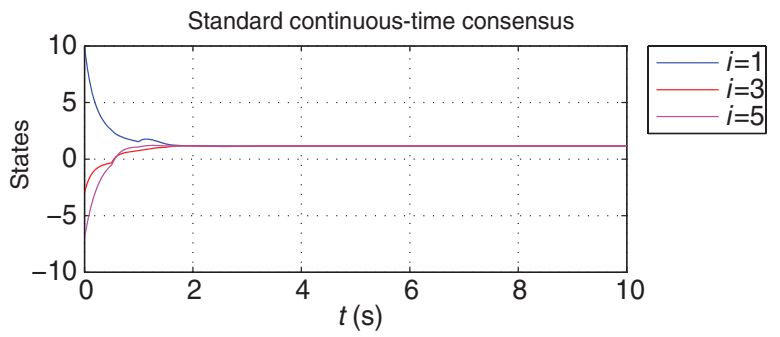

Continuous-time consensus with both the current and outdated states

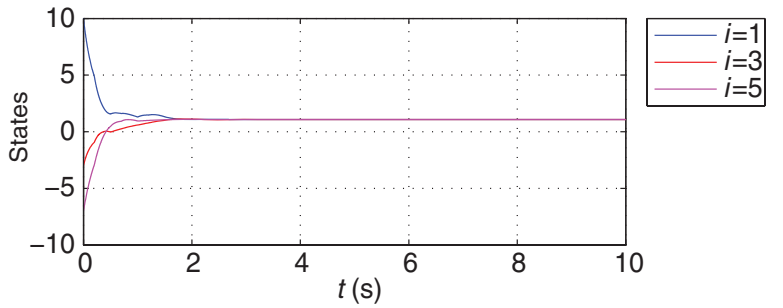

Figure 3. Convergence results using (15a) and (15b) under dynamic directed interaction.

$$
\begin{aligned}
\mathcal{L}_{2} & =\left(\begin{array}{cccccc}
3 & -1.5 & -1.5 & 0 & 0 & 0 \\
-2 & 2 & 0 & 0 & 0 & 0 \\
-1.5 & 0 & 4 & -1 & 0 & -1.5 \\
0 & -1 & 0 & 1 & 0 & 0 \\
0 & -1 & -1.5 & 0 & 3 & -0.5 \\
0 & 0 & 0 & -1 & 0 & 1
\end{array}\right), \\
\mathcal{L}_{3} & =\left(\begin{array}{cccccc}
4 & -4 & 0 & 0 & 0 & 0 \\
-1 & 2 & 0 & -1 & 0 & 0 \\
-1 & 0 & 3 & -1 & 0 & -1 \\
0 & 0 & -1 & 2 & -1 & 0 \\
0 & -1 & 0 & 0 & 1.5 & -0.5 \\
0 & 0 & -1 & -1 & 0 & 2
\end{array}\right), \\
\mathcal{L}_{4} & =\left(\begin{array}{cccccc}
3 & -1 & 0 & 0 & -2 & 0 \\
-1 & 1.5 & 0 & 0 & -0.5 & 0 \\
-1.5 & 0 & 2 & 0 & -0.5 & 0 \\
0 & 0 & 0 & 1 & 0 & -1 \\
0 & -0.5 & -1.5 & 0 & 2 & 0 \\
0 & -1 & -1 & 0 & -1 & 3
\end{array}\right),
\end{aligned}
$$

at time instants $\eta_{i}=0.5 i \mathrm{~s}, i=1,2, \ldots$ Figures 3 and 4 show the convergence results and control efforts of 

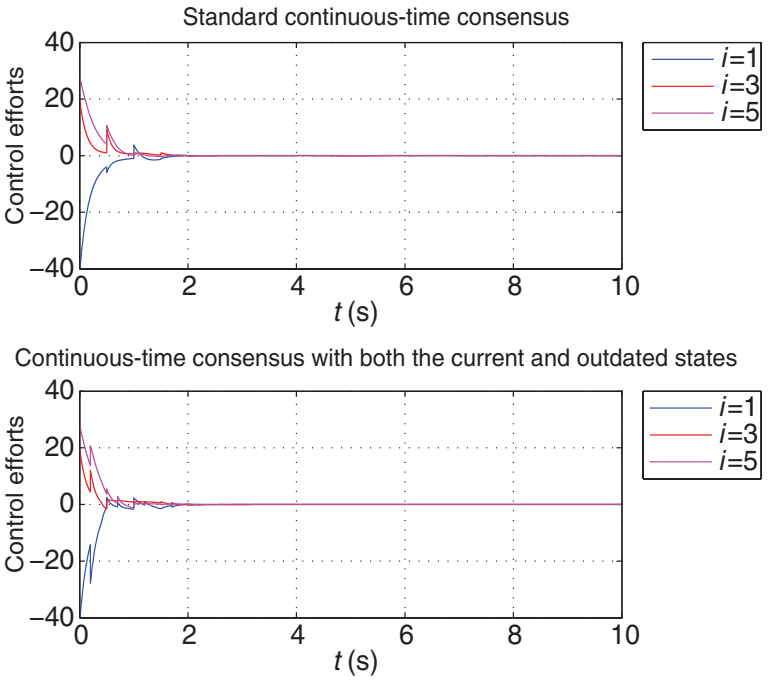

Figure 4. Control efforts using (15a) and (15b) under dynamic directed interaction.

Table 2. Convergence time comparison in the continuoustime case under dynamic directed interaction.

\begin{tabular}{lcc}
\hline & $\begin{array}{c}\text { Within 5\% } \\
\text { of final } \\
\text { equilibrium }\end{array}$ & $\begin{array}{c}\text { Within 2\% } \\
\text { of final } \\
\text { equilibrium }\end{array}$ \\
\hline System (10) using (15a) & $2.26 \mathrm{~s}$ & $2.87 \mathrm{~s}$ \\
$\begin{array}{l}\text { System (10) using (15b) } \\
\text { Convergence time }\end{array}$ & $2.19 \mathrm{~s}$ & $2.62 \mathrm{~s}$ \\
improvement percentage & 3 & 8.7 \\
\hline
\end{tabular}

system (10) using, respectively, (15a) and (15b) under dynamic directed interaction while Table 2 shows the convergence time using (15a) and (15b). Similar to the fixed communication graph case, the convergence speed of multi-agent consensus with both the current and outdated states is improved if $\tau$ is chosen properly.

In the discrete-time setting, for system (26) using (27a) and (27b), we choose $T=0.05 \mathrm{~s}, N=4$ and

$$
\mathcal{L}=\left(\begin{array}{cccccc}
2 & -1 & -1 & 0 & 0 & 0 \\
-1 & 1 & 0 & 0 & 0 & 0 \\
-1 & 0 & 3 & -1 & -1 & 0 \\
0 & 0 & -1 & 2 & -1 & 0 \\
0 & 0 & -1 & -1 & 3 & -1 \\
0 & 0 & 0 & 0 & -1 & 1
\end{array}\right)
$$

Figures 5 and 6 show the convergence results and control efforts of system (26) using, respectively, (27a) and (27b) while Table 3 shows the convergence time using (27a) and (27b). We can see that the convergence speed of discrete-time multi-agent consensus with both the current and outdated states is improved if $T$ and $N$

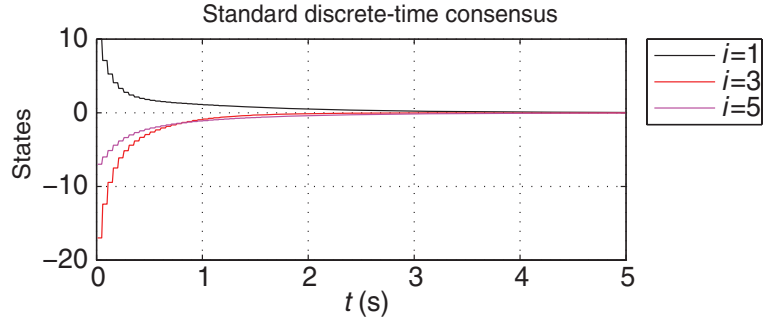

Discrete-time consensus with both the current and outdated states

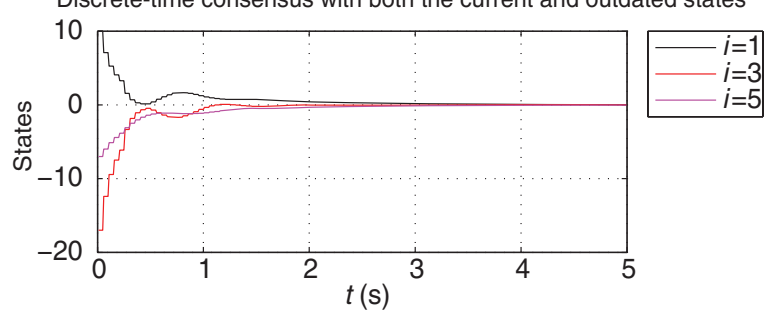

Figure 5. Convergence results using (27a) and (27b).
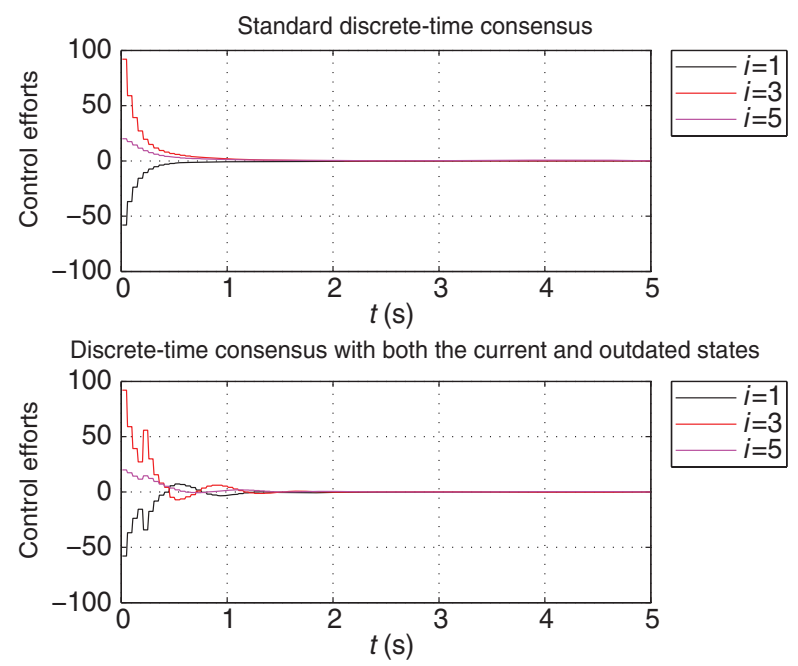

Figure 6. Control efforts using (27a) and (27b).

Table 3. Convergence time comparison in the discrete-time case.

\begin{tabular}{lcc}
\hline & $\begin{array}{c}\text { Within 5\% } \\
\text { of final } \\
\text { equilibrium }\end{array}$ & $\begin{array}{c}\text { Within 2\% } \\
\text { of final } \\
\text { equilibrium }\end{array}$ \\
\hline $\begin{array}{l}\text { System (26) using (27a) } \\
\text { System (26) using (27b) }\end{array}$ & $5.91 \mathrm{~s}$ & $6.46 \mathrm{~s}$ \\
$\begin{array}{l}\text { Convergence time } \\
\text { improvement percentage }\end{array}$ & $16.91 \mathrm{~s}$ & $5.41 \mathrm{~s}$ \\
\hline
\end{tabular}

are chosen properly under an undirected fixed communication graph. Also, the maximum control effort for the consensus algorithm with both the current and outdated states is identical to that for the standard one. 


\section{Concluding remarks and future work}

This article studied consensus algorithms using both the current and outdated states for continuous-time and discrete-time multi-agent systems. In the continuous-time case, we showed that the algorithm with both the current and outdated states can achieve a faster convergence speed than that using the standard one under the directed fixed or dynamic communication graphs. Unlike the undirected graph, the distribution of eigenvalues of the Laplacian matrix associated with a directed graph will play an important role for the algorithm with both the current and outdated states under directed communication graphs. In the discrete-time case, the algorithm with both the current and outdated states was shown to achieve consensus with a faster convergence speed than that using the standard one if the sampling period and the outdated state information are chosen properly under the undirected fixed communication graphs. Simulation results supported the effectiveness of the algorithms. One interesting future direction is the robustness analysis of the proposed consensus algorithms with both the current and outdated states in the presence of real information delays. Another interesting future direction is the study of consensus with information reuse under nonlinear couplings. Of course, in the case of nonlinear couplings, the analysis tools and the definition of the convergence speed used in this article are no longer valid. We expect that a Lyapunov approach combined with a more general definition of the convergence speed might be promising.

\section{Acknowledgements}

This work was supported by a National Science Foundation CAREER Award (ECCS-0748287). Z. Meng would like to acknowledge the Ministry of Education of PR China and China Scholarship Council (CSC) for financially supporting him as a visiting PhD student at Utah State University from September 2008 to September 2009.

\section{References}

Agaev, R., and Chebotarev, P. (2000), 'The Matrix of Maximum Out Forests of a Digraph and its Applications', Automation and Remote Control, 61, 1424-1450.

Agaev, R., and Chebotarev, P. (2005), 'On the Spectra of Nonsymmetric Laplacian Matrices', Linear Algebra and its Applications, 399, 157-178.

Cao, M., Spielman, D.A., and Yeh, E.M. (2006), 'Accelerated Gossip Algorithms for Distributed Computation', in Proceedings of the 44th Annual Allerton Conference, September Allerton House, UIUC, IL, USA, pp. 952-959.
Cao, Y., and Ren, W. (2009), 'Multi-agent Consensus using Both Current and Outdated States with Fixed and Undirected Interaction', Journal of Intelligent and Robotic Systems, DOI: 10.1007/s10846-009-9337-7.

Fax, J.A., and Murray, R.M. (2004), 'Information Flow and Cooperative Control of Vehicle Formations', IEEE Transactions on Automatic Control, 49, 1465-1476.

Ghosh, B., Muthukrishnan, S., and Schultz, M.H. (1998), 'First and Second-order Diffusive Methods for Rapid, Coarse, Distributed Load Balancing', Theory of Computing Systems, 31, 331-354.

Horn, R.A., and Johnson, C.R. (1985), Matrix Analysis, Cambridge: Cambridge University Press.

Jadbabaie, A., Lin, J., and Morse, A.S. (2003), 'Coordination of Groups of Mobile Autonomous Agents using Nearest Neighbor Rules', IEEE Transactions on Automatic Control, 48, 988-1001.

Kim, Y., and Mesbahi, M. (2006), 'On Maximizing the Second Smallest Eigenvalue of a State-dependent Graph Laplacian', IEEE Transactions on Automatic Control, 51, 116-120.

Lee, D., and Spong, M.W. (2006), 'Agreement with Nonuniform Information Delays, in Proceedings of the American Control Conference, June, Minneapolis, MN, pp. 756-761.

Lin, J., Morse, A.S., and Anderson, B.D.O. (2003), 'The Multi-agent Rendezvous Problem', in Proceedings of the IEEE Conference on Decision and Control, Maui, Hawaii, pp. $1508-1513$.

Moreau, L. (2004a), Stability of Continuous-time Distributed Consensus Algorithms, available at arXiv:math.OC/ 0409010v1, September.

Moreau, L. (2004b), 'Stability of Continuous-time Distributed Consensus Algorithms', in Proceedings of the IEEE Conference on Decision and Control, December, Paradise Island, Bahamas, pp. 3998-4003.

Moreau, L. (2005), 'Stability of Multi-agent Systems with Time-dependent Communication Links', IEEE Transactions on Automatic Control, 50, 169-182.

Olfati-Saber, R., and Murray, R.M. (2004), 'Consensus Problems in Networks of Agents with Switching Topology and Time-delays', IEEE Transactions on Automatic Control, 49, 1520-1533.

Peng, K., and Yang, Y. (2009), 'Leader-following Consensus Problem with a Varying-velocity Leader and Time-varying Delays', Physica A, 388, 193-208.

Ren, W. (2007), 'Distributed Attitude Alignment in Spacecraft Formation Flying', International Journal of Adaptive Control and Signal Processing, 21, 95-113.

Ren, W., and Atkins, E.M. (2007), 'Distributed Multi-vehicle Coordinated Control via Local Information Exchange', International Journal of Robust and Nonlinear Control, 17, 1002-1033.

Ren, W., and Beard, R.W. (2005), 'Consensus Seeking in Multiagent Systems under Dynamically Changing Interaction Topologies', IEEE Transactions on Automatic Control, 50, 655-661.

Ren, W., Beard, R.W., and Atkins, E.M. (2007), 'Information Consensus in Multivehicle Cooperative 
Control: Collective Group Behavior through Local Interaction', IEEE Control Systems Magazine, 27, 71-82.

Tian, Y.P., and Liu, C.L. (2008), 'Consensus of Multiagent Systems with Diverse Input and Communication Delays', IEEE Transactions on Automatic Control, 53, 2122-2128.

Vicsek, T., Czirok, A., Jacob, E.B., Cohen, I., and Schochet, O. (1995), 'Novel Type of Phase Transitions in a System of Self-driven Particles', Physical Review Letters, 75, 1226-1229.
Xiao, F., and Wang, L. (2008a), 'Asynchronous Consensus in Continuous-time Multi-agent Systems with Switching Topology and Time-varying Delays', IEEE Transactions on Automatic Control, 53, 1804-1816.

Xiao, F., and Wang, L. (2008b), 'Consensus Protocols for Discrete-time Multi-agent Systems with Time-varying Delays', Automatica, 44, 2577-2582.

Xiao, L., and Boyd, S. (2003), 'Fast Linear Iterations for Distributed Averaging', in Proceedings of the IEEE Conference on Decision and Control, December, Maui, Hawaii, pp. 4997-5002. 